\title{
Rapid evaluation of S-N curve for composite laminates on thermographic approach
}

\author{
Jia Huang ${ }^{1}$, Christian Garnier ${ }^{2,}$, Marie-Laetitia Pastor $^{1}$, and Xiaojing Gong ${ }^{1}$ \\ ${ }^{1}$ Univ. Toulouse, IUT Tarbes, ICA, 1 rue Lautréamont, F-65016 Tarbes, France \\ ${ }^{2}$ Univ. Toulouse, INP-ENIT, LGP, 47 avenue d'Azereix, F-65016 Tarbes, France
}

\begin{abstract}
A new fatigue life prediction model is developed for composite laminates based on both infrared thermographic data and stiffness degradation process. The fatigue limit is firstly determined by the improved thermographic method and then a two-parameter function is proposed to describe the normalized stiffness degradation of specimens subjected to various maximum loading stresses. These two parameters can be calibrated by the stiffness degradation data and stabilized temperature data. Thereafter, the calibrated model can be used to predict S-N curve. The proposed model is applied the experimental data from literature and the results show that the predicted S-N curve has a well agreement with the experimental one.
\end{abstract}

\section{Introduction}

Fatigue property of composite laminates receives more and more concerns since they are usually used in aerospace, automotive and marine load-bearing components. Investigating the fatigue performance $\mathrm{f}$ composite laminates by traditional methods is a costly and time-consuming process. Moreover, due to the rich diversity (different orientations, fiber ratio, nature of fibers and matrix, etc.) in composite laminates, rapid evaluation of their fatigue property is of great significance for choosing proper structure of laminates in component designing.

It is a common phenomenon that the plastic deformation of the material is accompanied by the generation of a large amount of heat. This inspires researchers to measure temperature change as an effective approach of studying fatigue behavior. With appearance of highspeed, high-resolution infrared thermography (IRT), the slight change of temperature during fatigue, which is due to the thermal-elastic-plastic effect, can be detected. Based on IRT data analysis, many criteria have been developed to rapidly determine fatigue limit and obtain S-N curves for metallic alloys [1-7]. However, those criteria may not be accurate any more for composite laminates because damage and failure mechanisms are different and more complicated (compared to metallic materials).

Unlike metals, the damage mechanism in composite laminates under cyclic loading usually includes matrix cracking, delamination and fiber breakage etc. In general, there are three distinctive damage stages during the damage accumulation process in composite laminates under fatigue loading [8]: matrix cracking and interface debonding (I), delamination (II), delamination growth and localized fiber breakage (III). Since the stage III corresponding to final fracture stage is short-period where unstable fracture occurs, the first two stages are usually used to estimate the residual life of laminates. It is well known that the stiffness degradation in fatigue loaded specimen has also three stages due to material damage [9], thus some of the damage accumulation model was developed based on stiffness degradation in the reference [10].

In the present paper, a new fatigue life prediction model by combining infrared thermographic data and damage accumulation process is developed. By applying the improved thermographic method which is recently proposed, the fatigue limit can be easily and rapidly obtained. This work tries to give an answer to the question: how to quickly determine fatigue resistance in term of S-N curve by using thermographic method? Herein, curve fitting method is used to estimate the value of threshold of stiffness under the load corresponding to fatigue limit. After that, damage evolution curves under different maximum loading stresses can be calculated. Then, a two-parameter damage accumulation model is proposed to characterize the damage evolution curves. One parameter characterizes the influence of temperature rising on damage accumulation speed and the other one is used to regulate amplitude of function. After parameter calibration with the experimental stiffness degradation and IRT data, the model can be used to predict S-N curve.

\section{IRT technique foundation}

Generally, fatigue behavior can be considered as an irreversible process of mechanical property degradation 
under cyclic loading. There are two main approaches in this irreversible process [11]: (i) a chemical-physical process, such as dislocation, chemical bond rupture, creep deformation, etc. (ii) a physical separation of the material, such as cracks, cavitations, etc. Both of those two approaches will cause heat release which is called intrinsic dissipation. By using infrared thermographic camera, the variation of temperature can be measured with high precision. A local heat conduction equation [12-14] can be employed here to explain the mechanism above:

$$
\rho C \dot{T}-\operatorname{div}(h \operatorname{grad} T)=d_{i}+s_{\text {the }}+s_{\text {ic }}+r_{e}
$$

where, $\rho, \mathrm{C}, \mathrm{T}, h$ are mass density, heat capacity, temperature and heat conduction coefficient, respectively. The first term $\rho C \dot{T}$ on the left side is the heat storage rate characterized by temperature change, and the second left-hand term $-\operatorname{div}(k \operatorname{grad} T)$ characterizes heat loss rate induced by conduction. The term group on the right side represents the different heat sources: $d_{i}$ denotes the intrinsic dissipation source; $s_{\text {the }}$ is the thermoelastic source; sic describes the heat source induced by the coupling effect between internal variables and temperature; and $r_{e}$ denotes the external heat supply. When components or specimens are under homogeneous uniaxial loading, we can have the following hypothesis:

(1) Parameters $\rho, \mathrm{C}, h$ are material constants and independent of the internal states.

(2) Thermoelastic source $S_{\text {the }}$ only causes small fluctuations of temperature but does not contribute to mean temperature rising [15].

(3) Internal coupling source between internal variables and temperature, sic, can be neglected [16].

(4) External heat supply re is time-independent and can be removed by using reference specimen placed nearby.

Based on the hypothesis above, the intrinsic energy dissipation $d_{i}$ can be identified as the main contributor to the total heat generated. Particularly, as can be seen from Eq. (1), if $d_{i}$ remains unchanged, with the increase of temperature, the heat loss rate, $-\operatorname{div}(h \operatorname{grad} T)$, will also increase. It can be deduced that when temperature $\mathrm{T}$ reaches a certain value, there will be a balance between heat loss and intrinsic energy dissipation. In this situation, the heat storage rate $\rho C \dot{T}$ equals to zero, so that $\mathrm{T}$ will remain stable. This conclusion has been proved by a number of experiments (with constant stress ratios) in the previous works [14, 17-20]. As shown in Fig. 1, there are three phases from the beginning of test to the final breakage of specimen. The temperature increases from initial value (room temperature) in phase I, then remains stable for the longest period in phase II and finally experiences a sharp increase just prior to the final breakage in phase III. Thus, it can be derived that $d_{i}$ keeps unchanged in phase II, which indicates that the damage accumulation rate is stable in this phase. If different loading stresses are applied, different stabilized temperature rising $\Delta T_{\text {stab }}$ will be obtained. Thus, the relationship of $\Delta T_{\text {stab }}$ and maximum applied loading stress $\sigma$ can be plotted, as shown in Fig. 1. Based on this, two straight lines are used to characterize the relationship and the intersection can be considered as fatigue limit $[11,21]$.

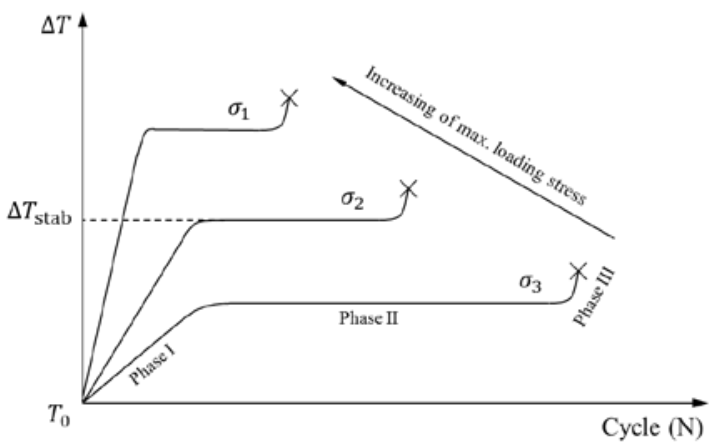

Fig. 1. Typical temperature evolution during fatigue test.

\section{Stiffness degradation model}

As known from literature, the main stiffness degradation process observed in composites laminates under fatigue loading can also be divided into three main stages $[8$, 10], as shown in Fig. 2. In stage 1, the normalized stiffness decreases rapidly with micro cracks initiation in the first few cycles and some fibers with low strength may break during this stage. Then the normalized stiffness experiences a slow and steady reduction mainly due to delamination and matrix crack propagation in stage 2. Prior to final breakage, the normalized stiffness drops down dramatically with a mass of fibers failure in stage 3 . It is commonly known that stage 3 are shortperiod and unstable so that the first two stages are usually used to estimate the residual life of laminates. Similar as the temperature evolution curves, the stage 2 with stable damage growth accounts for the most parts of the total cycle numbers. Therefore, we speculate that there is a certain relationship between $\Delta T_{\text {stab }}$ and the normalized stiffness degradation rate.

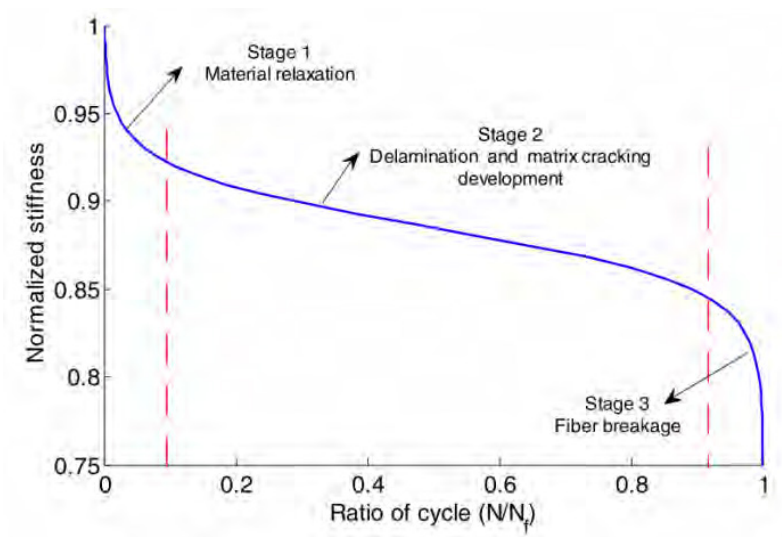

Fig. 2. Typical temperature evolution during fatigue test [9].

In order to propose a practical stiffness degradation model, two reasonable simplifications are made here. Firstly, only stage 1 and stage 2 are taken into account because stage 3 is short-period, unstable and difficult to capture [22, 23]. Secondly, for given composite laminates with same stacking sequence, ply orientation, geometry and fabrication process, if they are subjected to 
cyclic loading with fixed loading frequency and loading stress ratio, the critical values of normalized failure threshold stiffness $k_{f}$ can be considered to be independence of the maximum loading stress level. This has been proved by previous experimental work [9]. Thereafter, the normalized stiffness degradation model is proposed as:

$$
k(N)=1-p \Delta T_{\text {stab }} \ln \left(\frac{N}{q}+1\right)
$$

where $p$ and $q$ are two constant parameters which need to be calibrated by experimental data. It will be introduced in details in Section 4. The term $N^{1 / q}$ characterizes the shape of normalized stiffness with the increase cyclic number during stage 1 and stage 2. $\Delta T_{\text {stab }}$ is used to describe the general degradation speed, which is related to damage accumulation rate. In the proposed model, the normalized stiffness $k(N)$ starts from 1, decreases sharply and then becomes stable when cyclic number gets larger, which is similar as experimental normalized stiffness evolution. For Eq. (2), if cycle number $N$ is replaced by the final failure cycle number $N_{f}$, we can obtain:

$$
N_{f}=q\left(e^{\frac{1-k_{f}}{p \Delta T_{s t a b}}}-1\right)
$$

As can be observed from Eq. (3), for different stabilized temperatures we can obtain different failure cycle number $N_{f}$. And the different stabilized temperatures is depended on different maximum loading stress, as shown in Fig. 1. Thus, the new proposed model can predict the whole $\mathrm{S}-\mathrm{N}$ curve.

\section{Results and discussion}

The experimental data used in the present paper is taken from [18], where both IRT and stiffness degradation data are available, as shown in Fig. 3 and Fig. 4.

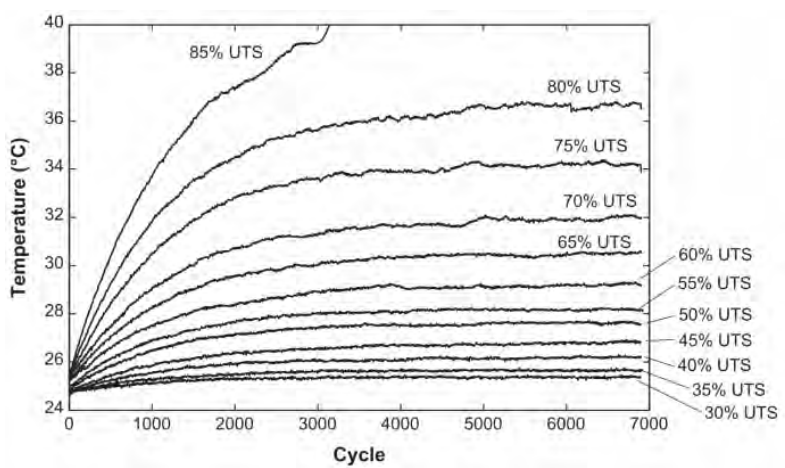

Fig. 3. Experimental IRT data taken from [18]. (UTS: Ultimate Tension Stress)

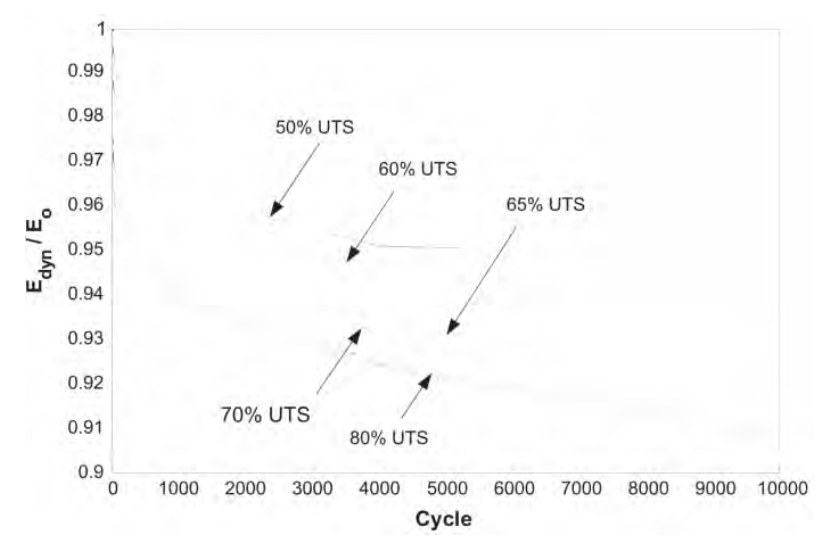

Fig. 4. Experimental normalized stiffness degradation data taken from [18].

The material tested in [18] is carbon fiber reinforced composite plate which is made from triaxially braided carbon fiber (T650/35-6 K) fabric with $0^{\circ} / \pm \theta$ braid orientation and thermosetting polyamide resin. The parameter calibration procedure and results are listed as below:

(1) Determine fatigue limit.

The improved thermographic methods in article [15] were firstly applied. There are three different improved graphic methods and we chose Method 2 and Method 3 here because for the IRT data in [18], the values of fatigue limit determined by Method 1 and Method 3 are the same, while Method 3 has a relative larger application scope. The fatigue limit was determined as $58 \%$ UTS by Method 2 and $67 \%$ UTS by Method 3, as shown in Fig. 5 and Fig. 6, respectively. The stabilized temperature rising corresponding to fatigue limit $\left(\Delta T_{\text {stab_fl }}\right)$ is also determined as $4.7{ }^{\circ} \mathrm{C}$ and $6.3{ }^{\circ} \mathrm{C}$, which will be used to determine normalized failure threshold stiffness $k_{f}$.

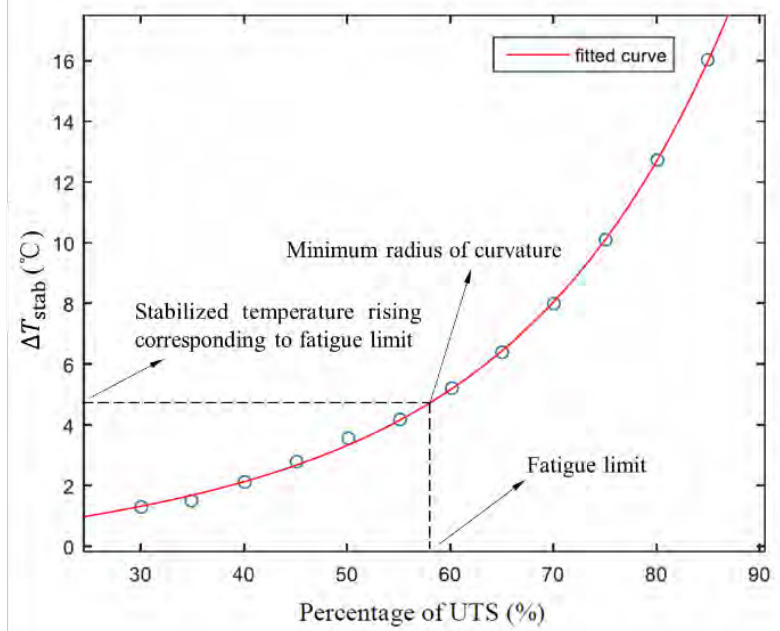

Fig. 5. Fatigue limit determined by Method 2. 


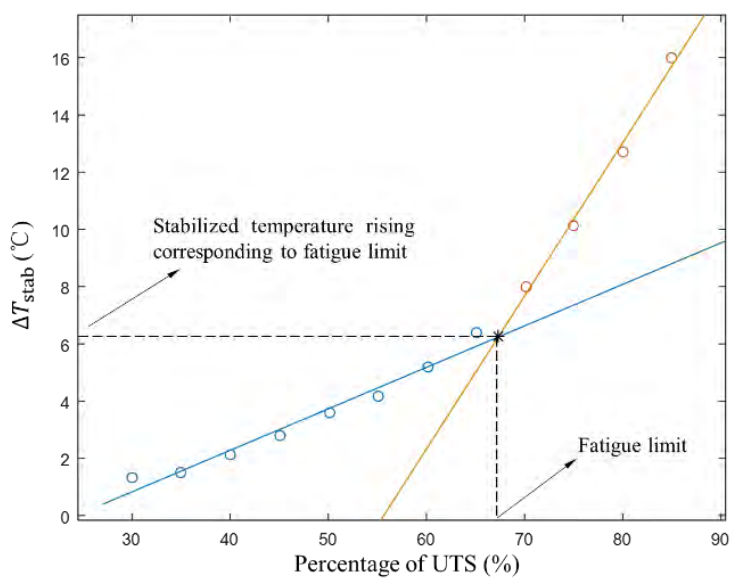

Fig. 6. Fatigue limit determined by Method 3.

(2) Determine the values of parameters $p$ and $q$. According to experimental normalized stiffness degradation data in Fig. 4, there are five different stiffness degradation curves. For each curve, 20 points were sampled, so a total of 100 points are used for fitting. Similarly, Eq. (2) is used to fit the data (3D surface fitting in MATLAB Toolbox) and the values of parameters $p$ and $q$ can be calibrated. The fitting results are shown in Fig. 7, and the values of $p$ and $q$ are determined as 0.001042 and 2.035 , respectively.

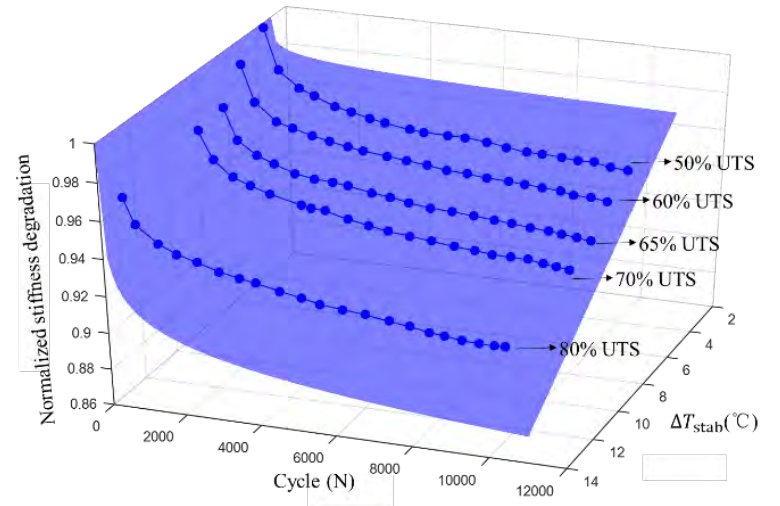

Fig. 7. Surface fitting of Eq. (3) by MATLAB.

(3) Calculate failure threshold stiffness.

Similar as previous case, The failure cycle number corresponding to fatigue limit, $N_{\mathrm{fl}}$, is usually taken between $10^{6}-10^{7}$. Herein, both the $k\left(N_{\mathrm{fl}}=10^{6}\right)$ and $k\left(N_{\mathrm{fl}}=10^{7}\right)$ are considered to calculate the failure threshold stiffness $k_{f}$. According to Eq. (2), we can obtain:

$$
k_{r}=1-p \Delta T_{\text {stab } \mathrm{f}} \ln \left(\frac{N_{\mathrm{f}}}{q}+1\right)
$$

Thus, $k_{f}$ corresponding to different values of $N_{\mathrm{fl}}$ and $\Delta T_{\text {stab_fl }}$ can be calculated.

(4) Calculate $S-N_{f}$ curve.

After knowing the values of $\mathrm{p}, \mathrm{q}$ and $k_{f}$, we can use Eq. (3) to calculate $S-N_{f}$ curve. So the relationship between $\Delta T_{\text {stab }}$ and maximum applied stress $\left(\sigma_{a}\right)$ should be established. For Method 2, the expression is given here:

$$
\Delta T_{\text {stab }}=a \cdot \exp \left(b \sigma-\frac{1}{b \sigma}\right)+c \sigma
$$

where the first term on right side is used to describe temperature response under high loading stress amplitude, and the second term on the right side is used to represent temperature rising under low loading stress amplitude. $a, b, c$ are three parameters of interpolation to be determined. $a$ is used to regulate the amplitude of exponential function, $b$ is used to adjust $\sigma_{a}$ axis range, and $c$ is used to describe the amplitude of temperature rising in low stress cases. By combining Eq. (3) and Eq. (5), we have:

$$
N_{f}=q e^{\frac{1-k_{f}}{p a \cdot \exp \left(b \sigma-\frac{1}{b \sigma}\right)+c \sigma}}-q
$$

By applying Eq. (6), the S-N curve can be predicted, as shown in Fig. 8.

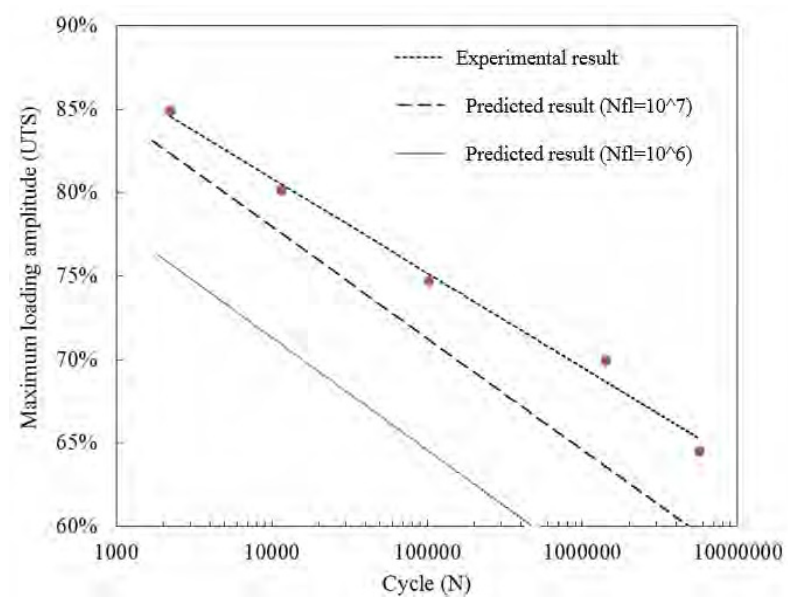

Fig. 8. Comparison of predicted results based on Method 2 and traditional test result.

As can been seen in Fig. 8, for triaxially braided CFRP laminates, the predicted $S-N_{f}$ curves corresponding to $N_{\mathrm{fl}}=10^{7}$ and $N_{\mathrm{fl}}=10^{6}$ are both lower than the experimental curve. The results indicate that although the S-N curve predicted by model relative to $N_{\mathrm{fl}}=10^{6}$ is very conservative and safe, the error between predicted result and experimental result is too large. So for model based on Method 2, only $N_{\mathrm{fl}}=10^{7}$ is recommended for engineering applications.

For Method 3, the relationship can be considered as two two-straight-line section function and the expression is given here:

$$
\Delta T_{\text {stab }}=\left\{\begin{array}{l}
a_{1} \sigma+b_{1}\left(-b_{1} / a_{1} \leq \sigma<\sigma_{\mathrm{f}}\right) \\
a_{2} \sigma+b_{2}\left(\sigma \geq \sigma_{\mathrm{f}}\right)
\end{array}\right.
$$

where $a_{1}, b_{1}, a_{2}$ and $b_{2}$ are four coefficients depending on those two straight lines. $\sigma_{\mathrm{fl}}$ is the value of maximum applied loading stress corresponding to fatigue limit. For engineering applications, we usually just need to know the fatigue life when the maximum loading stress is higher than fatigue limit, so we have

$$
N_{f}=q e^{\frac{1-k_{f}}{p\left(a_{2} \sigma+b_{2}\right)}}-q\left(\sigma \geq \sigma_{\mathrm{fl}}\right)
$$

By applying Eq. (8), the S-N curve can also be calculated, as shown in Fig. 9. 


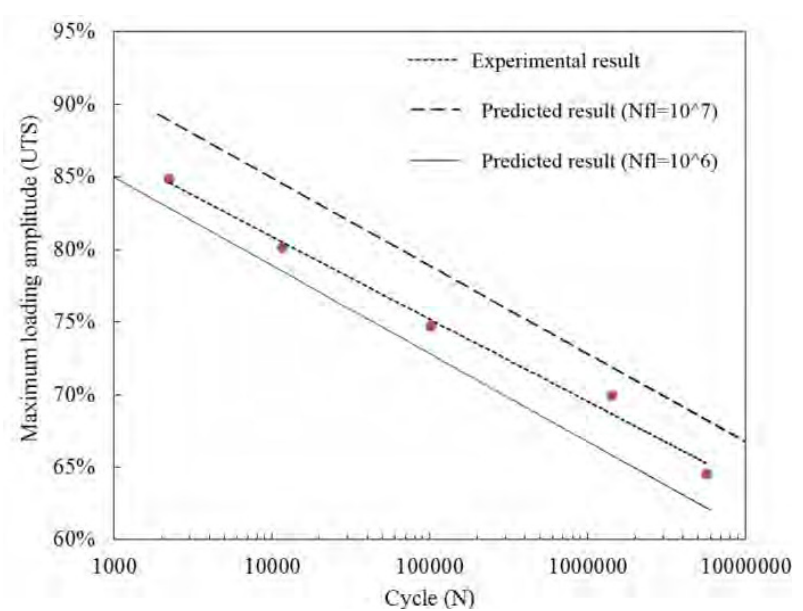

Fig. 9. Comparison of predicted results based on Method 3 and traditional test result.

As can be observed in Fig. 9, the predicted $S-N_{f}$ curve corresponding to $N_{\mathrm{fl}}=10^{7}$ is overall higher than the experimental curve and the error between predict value and experimental is relatively larger for low fatigue life (less than 10000 cycles). On the contrary, the predicted $S-N_{f}$ curve corresponding to $N_{\mathrm{fl}}=10^{6}$ is lower than the traditional test result. Therefore, due to safety reasons, for model based on Method $3, N_{\mathrm{fl}}=10^{6}$ is recommended here for engineering applications because the predicted result is more conservative.

\section{Conclusions}

The proposed model combined temperature rising with the stiffness degradation process. It was speculated that the stabilized temperature could reflect the rate of damage accumulation. To the authors' knowledge it is the first time both IRT and stiffness degradation data are taken into consideration to predicted fatigue behaviour of composites. A two-parameter model was developed to characterize stiffness degradation process. These two parameters were then calibrated by 3-D surface fitting method. Experimental validation shows that the new proposed model can predicted curves with good accuracy. The whole determination process takes about 10-12 hours, which is much faster than traditional fatigue test method. To sum up, this paper provides a quick and simple way to evaluate fatigue limit and S-N of composite laminates for engineering applications.

The author Jia Huang would like to acknowledge China Scholarship Council for the financial support of 3 years study at the University of Toulouse.

\section{References}

1. Huang, Y., S. Li, S. Lin, and C. Shih, Using the method of infrared sensing for monitoring fatigue process of metals. Mater. Eval. (United States), 42(8) (1984)

2. Jiang, L., H. Wang, P. Liaw, C. Brooks, and D. Klarstrom, Characterization of the temperature evolution during high-cycle fatigue of the ULTIMET superalloy: experiment and theoretical modeling. Metallurgical and Materials Transactions A, 32(9): 2279-2296 (2001)

3. Jiang, L., H. Wang, P. Liaw, C. Brooks, L. Chen, and D. Klarstrom, Temperature evolution and life prediction in fatigue of superalloys. Metallurgical and Materials Transactions A, 35(3): 839-848 (2004)

4. Yang, B., P. Liaw, G. Wang, M. Morrison, C. Liu, R. Buchanan, and Y. Yokoyama, In-situ thermographic observation of mechanical damage in bulk-metallic glasses during fatigue and tensile experiments. Intermetallics, 12(10): 1265-1274 (2004)

5. Curà, F., G. Curti, and R. Sesana, A new iteration method for the thermographic determination of fatigue limit in steels. International Journal of Fatigue, 27(4): 453-459 (2005)

6. Amiri, M. and M.M. Khonsari, Life prediction of metals undergoing fatigue load based on temperature evolution. Materials Science \& Engineering A, 527(6): 1555-1559 (2010)

7. Amiri, M. and M.M. Khonsari, Rapid determination of fatigue failure based on temperature evolution: Fully reversed bending load. International Journal of Fatigue, 32(2): 382-389 (2010)

8. Vasiukov, D., S. Panier, and A. Hachemi, Direct method for life prediction of fibre reinforced polymer composites based on kinematic of damage potential. International Journal of Fatigue, 70: 289296 (2015)

9. Peng, T., Y. Liu, A. Saxena, and K. Goebel, In-situ fatigue life prognosis for composite laminates based on stiffness degradation. Composite Structures, 132: 155-165 (2015)

10. Shiri, S., M. Yazdani, and M. Pourgol-Mohammad, A fatigue damage accumulation model based on stiffness degradation of composite materials. Materials \& Design, 88: 1290-1295 (2015)

11. Luong, M.P., Fatigue limit evaluation of metals using an infrared thermographic technique. Mechanics of materials, 28(1): 155-163 (1998)

12. Boulanger, T., A. Chrysochoos, C. Mabru, and A. Galtier, Calorimetric analysis of dissipative and thermoelastic effects associated with the fatigue behavior of steels. International Journal of Fatigue, 26(3): 221-229 (2004)

13. Chrysochoos, A., V. Dattoma, and B. Wattrisse, Deformation and dissipated energies for high cycle fatigue of 2024-T3 aluminium alloy. Theoretical \& Applied Fracture Mechanics, 52(2): 117-121 (2009)

14. Guo, Q., X. Guo, J. Fan, R. Syed, and C. Wu, An energy method for rapid evaluation of high-cycle fatigue parameters based on intrinsic dissipation. International Journal of Fatigue, 80: 136-144 (2015)

15. Huang, J., M.-L. Pastor, C. Garnier, and X. Gong, Rapid evaluation of fatigue limit on thermographic data analysis. International Journal of Fatigue, 104(Supplement C): 293-301 (2017)

16. Guo, Q. and X. Guo, Research on high-cycle fatigue behavior of FV520B stainless steel based on 
intrinsic dissipation. Materials \& Design, 90(Supplement C): 248-255 (2016)

17. La Rosa, G. and A. Risitano, Thermographic methodology for rapid determination of the fatigue limit of materials and mechanical components. International journal of fatigue, 22(1): 65-73 (2000)

18. Montesano, J., Z. Fawaz, and H. Bougherara, Use of infrared thermography to investigate the fatigue behavior of a carbon fiber reinforced polymer composite. Composite structures, 97: 76-83 (2013)

19. Wang, X.G., V. Crupi, X.L. Guo, and Y.G. Zhao, Quantitative thermographic methodology for fatigue assessment and stress measurement. International Journal of Fatigue, 32(12): 1970-1976 (2010)

20. Fargione, G., A. Geraci, G. La Rosa, and A. Risitano, Rapid determination of the fatigue curve by the thermographic method. International Journal of Fatigue, 24(1): 11-19 (2002)

21. Luong, M.P., Infrared thermographic scanning of fatigue in metals. Nuclear Engineering and Design, 158(2): 363-376 (1995)

22. Tao, C., H. Ji, J. Qiu, C. Zhang, Z. Wang, and W. Yao, Characterization of fatigue damages in composite laminates using Lamb wave velocity and prediction of residual life. Composite Structures, 166: 219-228 (2017)

23. Lee, L.J., K.E. Fu, and J.N. Yang, Prediction of fatigue damage and life for composite laminates under service loading spectra. Composites Science and Technology, 56(6): 635-648 (1996) 